\title{
Plasmodium serine hydroxymethyltransferase: indispensability and display of distinct localization
}

\author{
Wichai Pornthanakasem, Darin Kongkasuriyachai, Chairat Uthaipibull, Yongyuth Yuthavong \\ and Ubolsree Leartsakulpanich*
}

\begin{abstract}
Background: Serine hydroxymethyltransferase (SHMT), a pyridoxal phosphate-dependent enzyme, plays a vital role in the de novo pyrimidine biosynthesis pathway in malaria parasites. Two genes have been identified in Plasmodium spp. encoding a cytosolic SHMT (CSHMT) and putative mitochondria SHMT (MSHMT), but their roles have not been fully investigated.
\end{abstract}

Methods: The presence of Plasmodium SHMT isoforms in the intra-erythrocytic stage was assessed based on their gene expression using reverse transcription PCR (RT-PCR). Localization studies of Plasmodium SHMT isoforms were performed by transfection of fluorescent-tagged gene constructs into $P$. falciparum and expressions of fluorescent fusion proteins in parasites were observed using a laser scanning confocal microscope. Genetic targeting through homologous recombination was used to study the essentiality of SHMT in Plasmodium spp.

Results: Semi-quantitative RT-PCR revealed the expression of these two genes throughout intra-erythrocytic development. Localization studies using P. falciparum expressing fluorescent-tagged SHMT showed that PfCSHMTred fluorescent fusion protein (PfCSHMT-DsRed) is localized in the cytoplasm, while PfmSHMT-green fluorescent fusion protein (PfmSHMT-GFP) co-localized with Mitotracker ${ }^{\mathrm{TM}}$-labelled mitochondria as predicted. The essentiality of plasmodial CSHMT was inferred from transfection experiments where recovery of viable knock-out parasites was not achieved, unless complemented with a functional equivalent copy of shmt.

Conclusions: Distinct compartment localizations of PFSHMT were observed between cytoplasmic and mitochondrial isoforms, and evidence was provided for the indispensable role of plasmodial CSHMT indicating it as a valid target for development of novel anti-malarials.

Keywords: Plasmodium, Serine hydroxymethyltransferase, Localization

\section{Background}

The rapid emergence of resistance in Plasmodium falciparum to nearly all currently used anti-malarials makes control of falciparum malaria a difficult task. Identification of new drug targets for development of new antimalarials is urgently needed. The malaria parasite lacks thymidine salvage pathway and depends solely on de novo pyrimidine synthesis $[1,2]$, in contrast to the human host, which utilizes both de novo and salvage pathways. Serine hydroxymethyltransferase (SHMT) is one of three enzymes involved in dTMP cycle, namely,

\footnotetext{
* Correspondence: ubolsree@biotec.or.th

National Center for Genetic Engineering and Biotechnology, 113

Phahonyothin Road, Khlong Nueng, Khlong Luang, Pathum Thani 12120, Thailand
}

dihydrofolate reductase (DHFR) and thymidylate synthase (TS). SHMT has a pyridoxal phosphate as a cofactor and participates in one-carbon metabolism, in which SHMT converts serine and tetrahydrofolate (THF) to glycine and methylenetetrahydrofolate (MTHF) respectively. SHMT has been investigated as a possible drug target in cancer and microbial therapeutics, particularly as SHMT expression is tightly regulated with DNA replication during cell division and the enzyme catalyzes the rate-limiting step in dTMP synthesis cycle [3-9].

Two forms of SHMT, cytosolic (c) and mitochondrial $(\mathrm{m})$, can be found in eukaryotes [10,11]. Based on DNA sequence search in PlasmoDB, there are two genes encoding SHMT in Plasmodium spp.: Plasmodium falciparum contains PFL1720w (PF3D7_1235600), a 
previously characterized cSHMT gene (Pfcshmt), and PF14_0534 (PF3D7_1456100), a putative gene of mSHMT (Pfmshmt). While the enzymatic function of recombinant $P f \mathrm{cSHMT}$ has been shown, the heterologously expressed PfmSHMT was found to be inactive $[9,12,13]$. PfmSHMT has been proposed to function in association with glycine cleavage components [14], but experimental proof has yet to be provided. As for the cytosolic isoform, alignment of amino acid sequences of Plasmodium cSHMT with human cSHMT shows an overall $44 \%$ homology and $80 \%$ similarity at the active site. In contrast to mammalian SHMTs, Plasmodium SHMTs can convert D-serine, in addition to its physiological substrate L-serine, to glycine in the folatedependent reaction $[9,15]$. Comparison between the crystal structure of human cSHMT and homology model of $P f$ CSHMT has revealed differences at the substrate binding site, which could be exploited for the development of specific anti-malarial inhibitors that do not cross inhibit the human enzymes [16].

Despite several lines of indirect evidence for the essential role of SHMT in malaria parasite growth, there is hitherto a lack of direct demonstration of this notion. Here, the study provides the genetic evidence confirming the two distinct compartmental localization of SHMT isoforms and demonstrates the indispensable role of CSHMT in growth and development of Plasmodium parasites.

\section{Methods}

\section{Chemicals}

All chemicals used were of the highest quality commercially available. The sequences of primers are listed in Additional file 1.

\section{Semi-quantitative analysis of gene expression of SHMT isoforms in $P$. falciparum}

Semi-quantitative reverse transcription PCR (semiquantitative RT-PCR) was employed to measure expression levels of Pfcshmt (primers BglII 5'Pfcshmt and EcoRV $3^{\prime} P f c h m t$ ) and putative Pfmshmt (primers BglII $5^{\prime}$ Pfmshmt and KpnI $3^{\prime} P f m s h m t$ ) relative to that of house-keeping gene $P f \alpha$-tubulin-2 (primers Pfo-tubulin-2F and $P f \alpha-$ tubulin-2R). Total RNA was extracted from sorbitolsynchronized $P$. falciparum 3D7 strain at ring, early trophozoite, late trophozoite, and schizont stages using TRIzol $^{\circledR}$ reagent (Invitrogen ${ }^{\mathrm{TM}}$, California, USA). Contaminating DNA was removed with RNase-free DNase I (New England Biolabs, Massachusetts, USA). cDNA was synthesized using oligo-dT primer and M-MuLV reverse transcriptase (New England Biolabs). PCR amplification was conducted using $\mathrm{GoTaq}^{\circledR}$ DNA polymerase (Promega, Wisconsin, USA) and the following thermal cycling conditions: $95^{\circ} \mathrm{C}$ for 3 minutes; 20 or 25 cycles of $95^{\circ} \mathrm{C}$ for 30 seconds, $50^{\circ} \mathrm{C}$ for 30 seconds, and $72^{\circ} \mathrm{C}$ for 2 minutes; and a final heating step of $72^{\circ} \mathrm{C}$ for 5 minutes. Amplicons were resolved by $2 \%$ agarose gelelectrophoresis, stained with ethidium bromide, and analysed for their intensities with ImageQuant ${ }^{\mathrm{TM}}$ Software (Molecular Dynamics, California, USA).

\section{Plasmid constructions}

Plasmids for the study of gene knockout in Plasmodium berghei ANKA strain were constructed based on the sequence of pL0017 vector (The Malaria Research and Reference Reagent Resource Center; MR4), which contains Toxoplasma gondii dihydrofolate reductasethymidylate synthase (Tgdhfr/ts) and green fluorescent protein gene $(g f p)$ expression cassettes for pyrimethamine (PYR) selection and fluorescence detection of transfected parasites. The 553 and 1,018 bp of PCR amplicons, corresponding to $5^{\prime}$ - and $3^{\prime} U T R$ of Pbcshmt (PBANKA_145020) respectively, were produced initially from $P$. berghei genomic DNA (gDNA). The 5'UTR fragment was inserted into pL0017 at HindIII site, while the $3^{\prime} U T R$ fragment was inserted at KpnI and SacII sites respectively. This construct, pL0017_Ashmt, was used in the knockout study. For allelic replacement construct, gfp in pL0017_sshmt was replaced with Plasmodium vivax cshmt (Pvcshmt; PVX_100730) and named pL0017_(Pv) $\Delta$ shmt.

Vectors for localization study were modified from the original pSSPF2/PfHsp60-GFP vector (a gift from Shigeharu Sato, MRC National Institute for Medical Research, UK) [17]. Initially, a short linker encoding 14 amino acids (SASKLGTSRATNNT) was inserted at AvrII restriction site using two complementary oligonucleotides (Linker $\mathrm{F}$ and Linker $\mathrm{R}$ ), which resulted in pSSPF2/ PfHsp60-GFP-Link vector. In order to determine the subcellular localization of $P f \mathrm{cSHMT}$ in malaria parasite, $g f p$ in pSSPF2/PfHsp60-GFP-Link was replaced with the gene encoding red fluorescent protein DsRed generating pSSPF2/PfHsp60-DsRed. Then, the coding sequence of Pfcshmt was PCR amplified from cDNA and inserted into pSSPF2/PfHsp60-DsRed replacing a mitochondrial targeting sequence of PfHsp60 at BglII and KpnI sites. For construction of the vector to enable study of putative PfmSHMT localization, human dhfr in pSSPF2/PfHsp60GFP-Link was replaced with blasticidin $S$ deaminase (bsd) at BamHI and HindIII sites, after which the open reading frame region of putative Pfmshmt was inserted at $B g l \mathrm{II}$ and KpnI sites. DNA sequences of the two constructs, named pRL_PfCSHMT and pGL_PfmSHMT, were confirmed by DNA sequencing (1st BASE, Singapore).

\section{Parasite culture and transfection}

All animal experiments were performed according to the international and national guidelines for ethical conduct 
on the care and humane use of animals with approval of the Ethical Committee on Animal Experimentation, National Center for Genetic Engineering and Biotechnology (BIOTEC), Thailand. Mouse strain ICR was intraperitoneally infected with $P$. berghei $\left(10^{6}\right.$ infected (i) RBC), and blood from tail vein was collected for determining parasitaemia.

Transfection of plasmids into $P$. berghei was performed according to a previously described protocol [18]. In brief, 5-10 $\mu \mathrm{g}$ of each construct were linearized by digestion with SacII, and transfected into purified schizonts using Basic Parasite Nucleofector Kit 2 (Lonza AG, Cologne, Germany) and Amaxa Nucleofector ${ }^{\text {TM }}$ device (Amaxa Biosystems $\mathrm{GmbH}$, Cologne, Germany) according to pre-set U033 program. Transfected parasites then intravenously injected into mice tail vein and selected by providing the mice with $70 \mu \mathrm{g} / \mathrm{ml}$ PYR (Sigma-Aldrich, Missouri, USA) in drinking water.

For localization studies, P. falciparum strain 3D7 was cultured in human RBC (5\% haematocrit) in RPMI-1640 medium (Invitrogen ${ }^{\mathrm{TM}}$ ) supplemented with $0.3 \mathrm{~g} / \mathrm{l} \mathrm{L-glu-}$ tamine, $5 \mathrm{~g} / \mathrm{l}$ hypoxanthine and $10 \%$ human serum under an atmosphere of $1 \% \mathrm{O}_{2}$ and $5 \% \mathrm{CO}_{2}$ [19]. Transient transfection of $P$. falciparum with plasmids was performed by electroporation as previously described [20]. In short, 5-10\% synchronous ring stage parasites were electroporated with $100 \mu \mathrm{g}$ of plasmid using Gene Pulser Xcell Electroporation System (Bio-Rad Laboratories, California, USA) at $0.310 \mathrm{kV}$ and $950 \mu \mathrm{F}$. pRL_PfcSHMT and pGL_PfmSHMT transfected parasites were cultured in the presence of $2 \mathrm{nM}$ WR99210 and $2 \mu \mathrm{g} / \mathrm{ml}$ blastici$\operatorname{din} \mathrm{S}\left(\right.$ Invitrogen $^{\mathrm{TM}}{ }^{\text {) }}$ respectively.

\section{Molecular characterization of transfected $P$. berghei}

Blood from transfected $P$. berghei-infected mice was collected by heart puncture. White blood cells were removed by passage through a syringe packed with Whatman ${ }^{\circledR}$ CF11 cellulose powder. gDNA was extracted from intra-erythrocytic transfected parasites using Genomic DNA Mini Kit (Geneaid Biotech, Taiwan), and was used in PCR and Southern blotting to assess integration of plasmid constructs at the desired loci. Diagnostic PCR to amplify endogenous Pbcshmt, $5^{\prime}$ integration fragment, and $3^{\prime}$ integration fragment was performed using primer pairs of XhoI Pbcshmt F and BamHI Pbcshmt R, 5'UTR int Pbcshmt F and 5'UTR int Pbdhfr-ts R, and $3^{\prime}$ intDS F and 3'UTR int Pbcshmt R, respectively. The protocols described above were conducted also with wild type parasites. The putative Pbmshmt was amplified as a control (using XhoI putative Pbmshmt F and BamHI putative Pbmshmt $\mathrm{R}$ primers).

For Southern blot hybridization, approximately $20 \mu \mathrm{g}$ of gDNA extracted from transgenic and wild type parasites were digested with EcoRV and BglII. DNA fragments were separated by $1 \%$ agarose gel-electrophoresis and transferred to nylon membrane (Merck Milipore, Massachusetts, USA) for hybridization with digoxigenin-labelled $5^{\prime} \mathrm{UTR}$ and $3^{\prime} \mathrm{UTR}$ probes of Pbcshmt according to the manufacturer's protocol (DIG High Prime DNA Labeling and Detection Kit II; Invitrogen ${ }^{\mathrm{TM}}$ ).

Expression of Pbcshmt, putative Pbmshmt, Pvcshmt, and $P b \alpha$-tubulin-2 in wild type and transgenic $P$. berghei parasites were assessed by RT-PCR. Expression level of putative Pbmshmt was measured by quantitative RT-PCR (qRT-PCR) using CFX96 ${ }^{\mathrm{TM}}$ Real-Time System and $\mathrm{iQ}^{\mathrm{TM}}$ SYBR $^{\circledR}$ Green Supermix (Bio-Rad Laboratories) normalized to Pbo-tubulin-2 expression level Relative gene expression using $2^{-\Delta \Delta C T}$ formula. Total RNA and first strand cDNA were prepared for analysis by RT-PCR and qRT-PCR as described above.

\section{Parasite growth study}

Three ICR mice per group were injected intravenously in the tail vein with either wild type or transgenic parasites $\left(10^{6} \mathrm{iRBC} / \mathrm{mice}\right)$. Parasite numbers were counted every day using Giemsa-stained blood smears (from tail vein) under a light microscope.

\section{Fluorescence microscopy}

Parasites were stained with Mitotracker ${ }^{\mathrm{TM}}$ (Roche, Basel, Switzerland) and Hoechst 33258 (Sigma-Aldrich) dyes according to manufacturer's protocols. Localization of fluorescent protein-tagged SHMT isoforms in transfected parasites was determined using Zeiss LSM 700 laser scanning confocal microscope (Carl Zeiss MicroImaging $\mathrm{GmbH}$, Germany) at excitation and emission wavelengths of $555 \mathrm{~nm}$ and $572 \mathrm{~nm}$ respectively for DsRed, and at $488 \mathrm{~nm}$ and $509 \mathrm{~nm}$ respectively for GFP. Images were processed using ZEN 2009 software.

\section{Results}

\section{Expression pattern of PfSHMT}

Although $P f$ CSHMT has been characterized in a number of reports $[8,9,12,13,21]$, not much is known regarding the properties of putative PfmSHMT, except for its mitochondrial location based on gene prediction and annotation in PlasmoDB. When the expression patterns of the two shmt forms were compared in P. falciparum during intra-erythrocytic developmental stages using semiquantitative RT-PCR (normalized to that of Pfo-tubulin2 expression), transcripts of both Pfshmt forms were detected throughout all erythrocytic stages, with peak transcript levels appearing during early and late trophozoite stage for putative Pfmshmt and Pfcshmt respectively (Figure 1). These results are in agreement with the role of SHMT in dTMP biosynthesis required for DNA replication. 


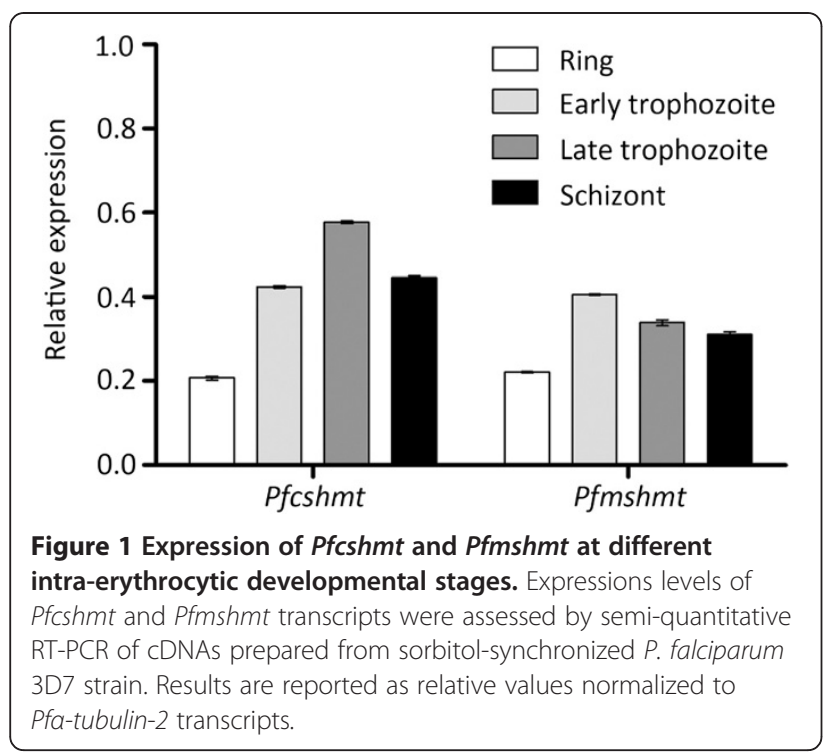

Localization of SHMT isoforms in P. falciparum

In order to identify the locations of the two PfSHMT isoforms in the parasite, pRL_PfCSHMT and pGL_PfmSHMT plasmids were constructed to express PfcSHMT fused with DsRed and PfmSHMT fused with GFP in the parasites (Figures $2 \mathrm{~B}$ and $2 \mathrm{C}$ ). The expression of these fusion proteins is driven by the constitutive promoter, PfHsp86
[17,22,23]. Transgenic parasites expressing GFP alone showed a diffuse distribution pattern of green fluorescence throughout the cytoplasm (Figure 2A). The distribution of DsRed-tagged $P f \mathrm{cSHMT}$ appears to be dominantly in cytoplasm (Figure 2B). PfcSHMT-DsRed fluorescence appears more variable in intensity compared to that of GFP alone in the cytoplasm. This may be partly explained by the superior fluorescence properties of GFP which is more intense and photostable compared to those of DsRed [24]. The intensity of the fluorescence reporter protein may also be affected when fused with another protein. The distribution of GFP-tagged PfmSHMT was colocalized with Mitotracker ${ }^{\mathrm{TM}}$ within the mitochondria (Figure 2C). The micrograph also suggest less intense signal seen in the area conterminous with the food vacuole, which likely comes from auto-fluorescence of crystalline haemozoin. This phenomenon was also observed in non-transfected parasites, especially when the image brightness was enhanced.

In most eukaryotes, a mitochondrial localization signal sequence is located at the $\mathrm{N}$-terminus of proteins targeted to this organelle [25]. However, this feature is not well-characterized in Plasmodium. PlasMit program [26] is the only available tool to predict Plasmodium mitochondrial-targeted proteins based on previously characterized malaria parasite mitochondrial proteins,

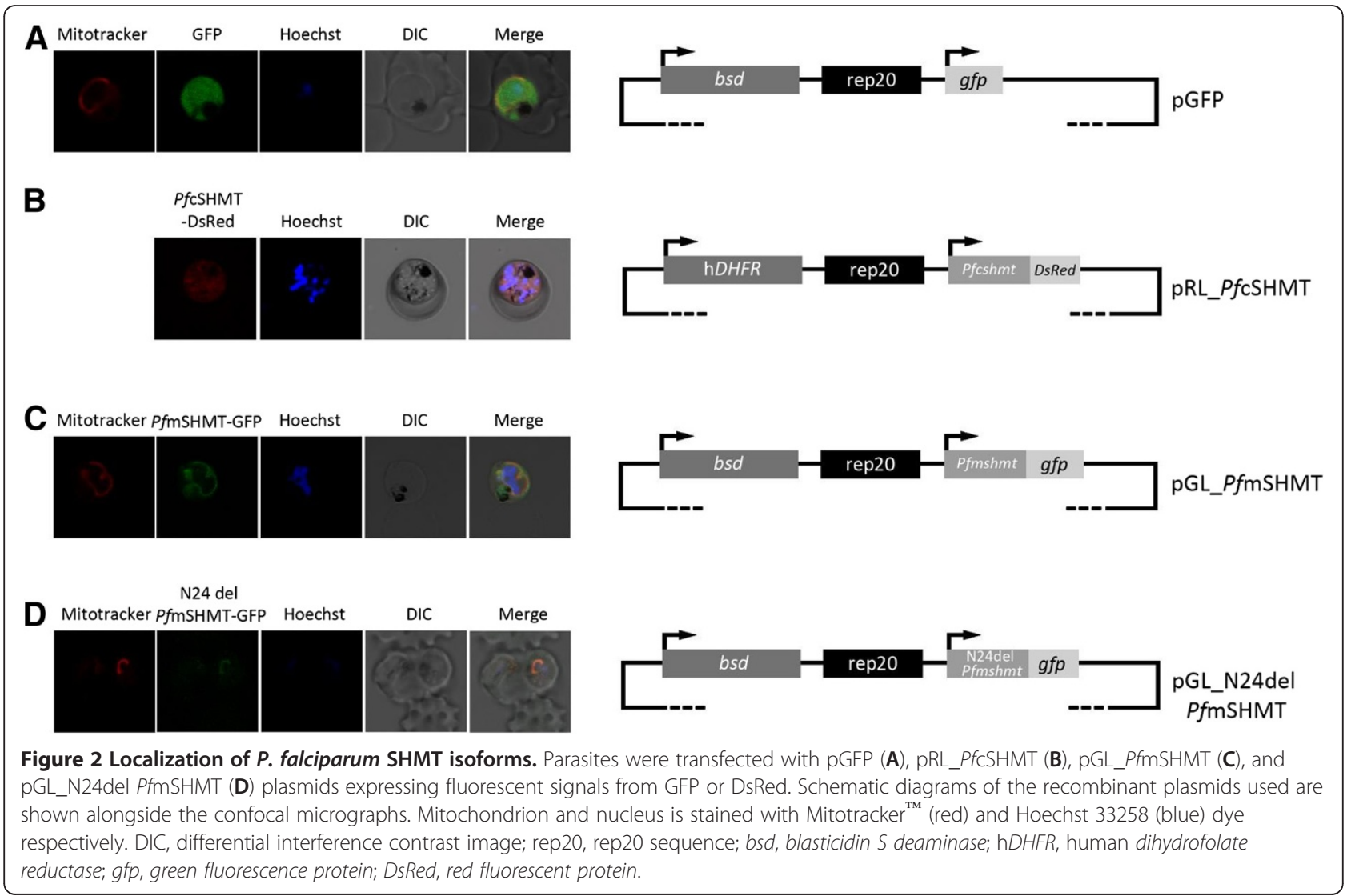


which analyzed the first $24 \mathrm{~N}$-terminal amino acids of PfmSHMT and predicted it to be a mitochondrial protein. Plasmid pGL_N24del PfmSHMT, expressing GFPtagged PfmSHMT with deletion of the first $24 \mathrm{~N}$ terminal amino acids (N-24 truncated PfmSHMT-GFP), was constructed to test the validity of this sequence as mitochondrial targeting signal. Confocal microscopy showed co-localization of N-24 truncated-PfmSHMTGFP with Mitotracker ${ }^{\mathrm{TM}}$ (Figure 2D), indicating that this $24 \mathrm{~N}$-terminal amino acid sequence does not play a role as a mitochondria targeting signal.

Recently, Read et al. [27] reported that the first $100 \mathrm{~N}$ terminal amino acids of PfmSHMT targets GFP-fusion protein to the mitochondrion. In order to identify the minimal mitochondrion-targeting sequence, plasmids were constructed to express GFP fusion proteins containing a series of truncations of the N-terminal 120 amino acids (Figure 3). All sequence-tagged-GFP were found only in the cytoplasm, except N1-80-GFP that was present in both mitochondrion and cytoplasm. Along with these results, in a separate experiment, the truncated del-N1-80-PfmSHMT-GFP was found in cytoplasm. The data suggest that the mitochondrial signal sequence is likely to involve longer span of PfmSHMT $\mathrm{N}$-terminal amino acids than previously predicted.

\section{Role of cSHMT in Plasmodium erythrocytic stages}

The role of Plasmodium cSHMT was assessed using $P$. berghei transfection system because genetic manipulations of $P$. berghei are more efficient than that of $P$. falciparum [28]. Two transfection plasmids were constructed, which upon double crossing-over recombination event from transfecting with linearized plasmids, resulted in replacement of Pbcshmt with the coding sequence either Pvcshmt or gfp (Figure 4A). Following selection of transfected P. berghei with PYR, only transgenic parasites containing Pvcshmt in place of Pbcshmt were recovered $(\triangle P b P v c s h m t)$. These results were consistent in three independent experiments. DNA analysis of transgenic parasite clones (obtained by limiting dilution) was undertaken to confirm the presence of the transgene, Pvcshmt. PCR amplification using specific primers for the recombination events in transgenic parasites showed a set of unique bands indicative of the integration of Pvcshmt (Figure 4B). Southern blot analysis indicated an absence of endogenous Pbcshmt in Pvcshmt replacement clones

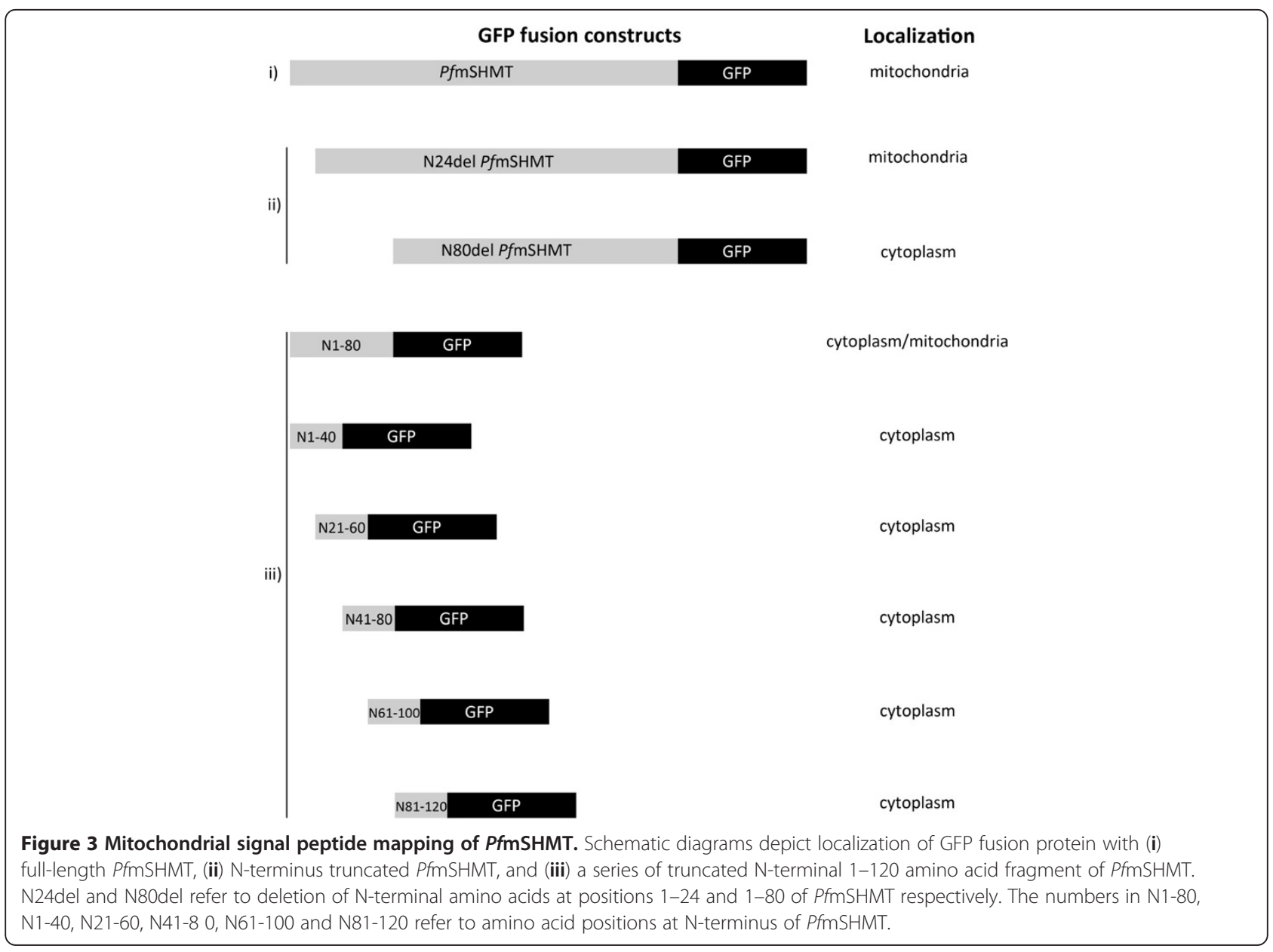




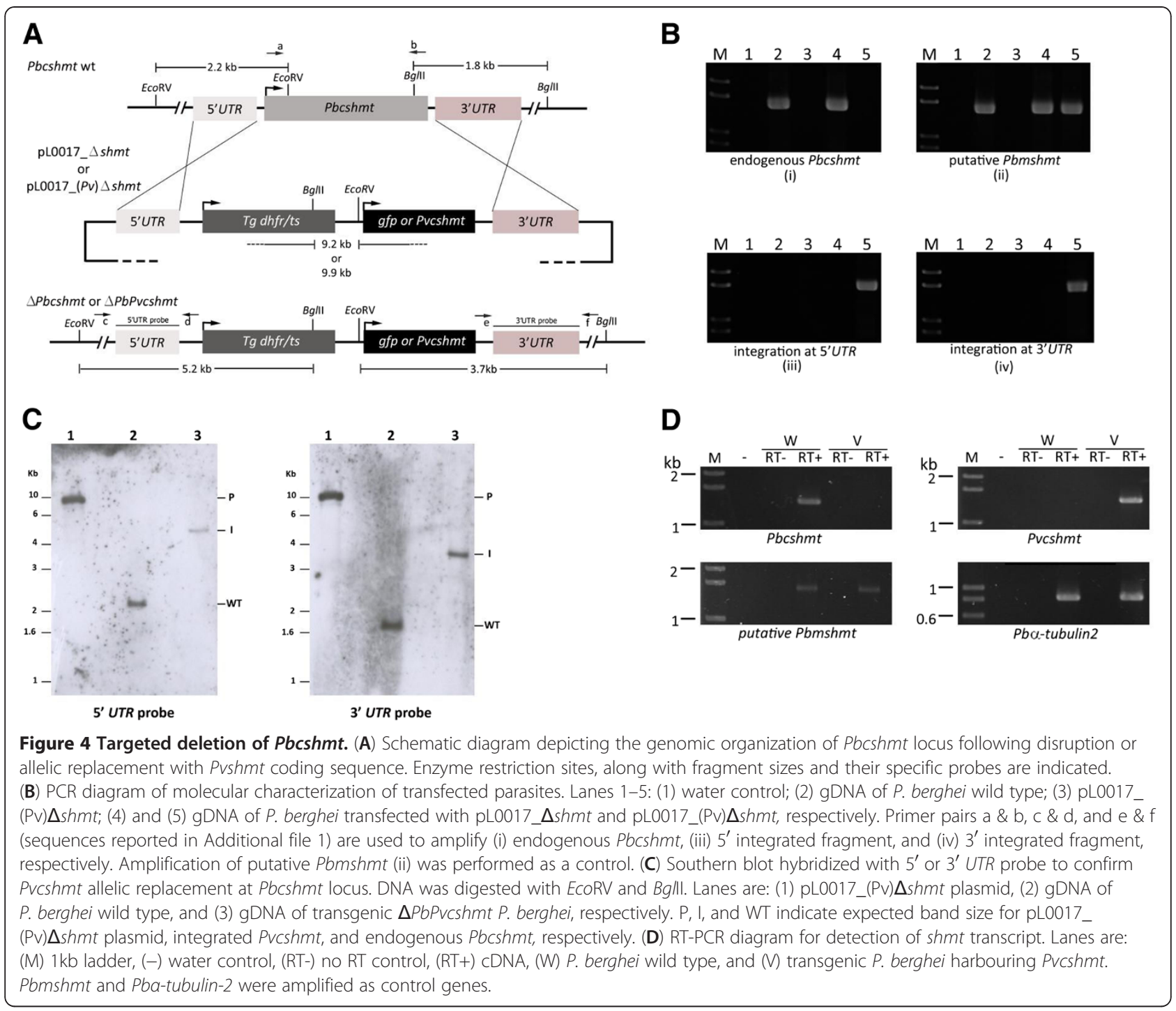

(Figure 4C). In addition, RT-PCR confirmed the absence of Pbcshmt expression, but that of Pvcshmt transcript instead (Figure 4D). These results rule out the recombination refractory property of the Pbcshmt locus and lend support to the indispensability of shmt for malaria parasite growth and development.

\section{Expression level of shmt in transgenic rodent malaria parasites and effect on growth rate}

In order to examine if the presence of Pvcshmt in transgenic $P$. berghei is functionally equivalent to that of endogenous Pbcshmt, growth profiles of these parasites were compared. Morphology of transgenic $\triangle P b P v c s h m t$ parasites at different sampling times did not appear to be affected (Figure 5A). However, the parasitaemia of transgenic $\triangle P b P v c s h m t$ parasites appeared to be less than that of the wild type (Figure 5B).
A redundant role for the two SHMT isoforms has been demonstrated in eukaryotes [29]. On the other hand, attempts in this study to knock out cshmt from both P. falciparum and P. berghei, and leaving mshmt intact, were unsuccessful. Expression profiles of mshmt in wild type and transgenic $\triangle P b P v c s h m t$ parasites were comparable. Thus, it does not appear that there is a redundancy role between plasmodial c- and mSHMT.

\section{Discussion}

SHMT links together several metabolic pathways, including biosynthesis of folate, dTMP, and methionine. The biological necessity of this enzyme in malaria parasites has been proposed as shmt transcripts are markedly increased during the rapid intra-erythrocytic stage progression [8]. Similar to other eukaryotes, Plasmodium spp. has two SHMT isoforms, a functioning cshmt and a putative mshmt allele. It is worth noting that, unlike 

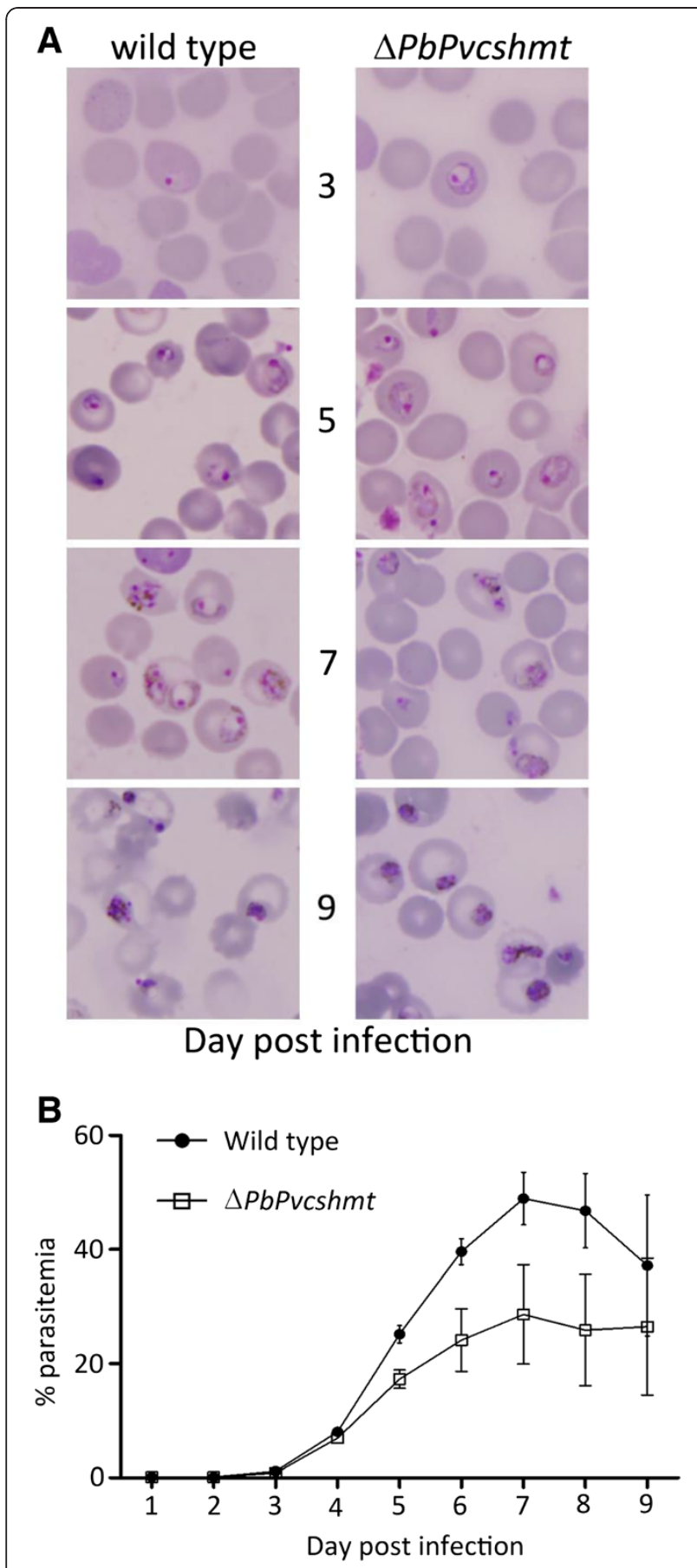

Figure $\mathbf{5}$ Morphology and parasite count. Morphology (A) and parasite count (B) of wild type and $\triangle P b P v c s h m t$ (allelic replacement with Pvshmt coding sequence) transgenic $P$. berghei. Thin blood smear and parasitaemia determination were performed every day post infection for 9 days.

other eukaryotes where c- and m-SHMT isozymes are highly conserved, $P f \mathrm{cSHMT}$ and $\mathrm{PfmSHMT}$ share only $\sim 20 \%$ similarity with each other [30].

Here, results show that Pfmshmt is a functional gene by demonstrating the expression of gene product throughout the asexual stage development. The presence of two isoforms in Plasmodium spp. raises the possibility of a redundant role and a potential overlap in their functional activity. For instance, in mice, examination of nuclear extracts of cshmt-knockout mice showed 25\% SHMT activity compared to wild type mice; the remaining SHMT activity is due to the presence of $\mathrm{mSHMT}$ in the nuclear extract, suggesting a redundant function of the two murine SHMT isoforms [29]. In the case of Plasmodium spp., null-mutants of cshmt-knockout parasite clones could not be recovered from transfected $P$. falciparum, even though methionine, folinic acid, or a mixture of these compounds was supplemented at concentrations 10-fold higher than that present in RPMI. However, in P. berghei, cshmt-knockout parasite clones could only be recovered when complemented with cshmt from another Plasmodium species (in this case $P$. vivax). These results provide experimental confirmation of the essentiality of cshmt in the survival of malaria parasites. In addition, these results suggest that there is functional conservation of cSHMT among Plasmodium spp., but not between cSHMT and mSHMT of the same species. On-going efforts to express recombinant $\mathrm{mSHMT}$ are in progress in order to confirm its role in malaria parasites.

The first $24 \mathrm{~N}$-terminal amino acids of putative plasmodial mSHMT contain several basic amino acids characteristics of mitochondrial targeting sequence [26,31]. Previous study observed that the first $100 \mathrm{~N}$-terminus of PfmSHMT is sufficient for mitochondria targeting [27]. In this study, transfection system using GFP reporter gene was taken to examine the cellular localization of PfmSHMT and to identify the minimum sequence required for mitochondrial targeting of this enzyme. Contrary to the previous prediction, the removal of putative mitochondria signal sequence $(\mathrm{N}$-terminus amino acids 1-24) of PfmSHMT did not affect its localization to the mitochondria, suggesting that that the targeting sequence may be downstream of the putative mitochondria targeting sequence. Systematic deletions of the first 120 amino acids of PfmSHMT demonstrated that the minimum leader sequence for mitochondrial targeting lies between amino acids 25-80. However, detection of cytoplasmic/mitochondria fluorescence of N1-80-GFP suggests that a more complex mechanism may be involved, such that a longer signal sequence may provide more specific localization to the mitochondria.

Intracellular localizations of $P f \mathrm{cSHMT}$ and $P f \mathrm{mSHMT}$ were addressed in this study by direct observation of SHMTs fusion with reporter protein compared to previously published work using immunofluorescence approach (IFA) [27]. The IFA with polyclonal antibody suggested a stage dependent localization pattern where PfCSHMT appeared in the cytoplasm, and also to 
apicoplast in the mid/late trophozoite to schizont stage. PfmSHMT appeared mainly in the mitochondria with some distribution in the cytoplasm. Multi-organelle localizations observed in these IFA experiments may be in part due to cross-reaction of polyclonal antibodies. Whilst the current work relies upon the intrinsic fluorescence from GFP or DsRed fused to SHMT of interest, with the assumption that the fusion proteins behave the same as native SHMTs. Despite different approaches, these studies are complementary of each other, as both studies revealed distinct compartment localization of $P f \mathrm{cSHMT}$ and PfmSHMT.

Various phenotypic consequences in shmt-deficient cells have been described. Inactivation of shmt results in glycine auxotroph phenotype in some organisms, such as Escherichia coli [32], while shmt mutations in Caenorhabditis elegans lead to maternal-effect lethal phenotype [33], pointing to the essential role of SHMT. In this study, attempts were made to generate Pbcshmt null mutant but the gene could not be replaced by a knockout construct. The refractoriness of Pbcshmt locus was ruled out as our attempts to replace the endogenous gene with Pvcshmt were successful. Additionally, the redundancy role of SHMTs in malaria parasite can be excluded. Transgenic $P$. berghei parasites containing Pvcshmt were able to infect murine red blood cells and complete their blood stage life cycle, albeit at a lower parasitaemia when compared with that of the wild type parasites. This implies that replacement of shmt affects fitness of transgenic parasite, which may be due to differences in catalytic efficiency between rodent and human plasmodial enzymes. This could readily be proven by comparing kinetic parameters of recombinant $P b c S H M T$ and $P v c S H M T$. It should also be noted that the expression of PvcSHMT was regulated by Pbeef $1 \alpha$ promoter, which might have an effect on the growth of mutant parasite.

Plasmodium SHMT has been suggested to be the ratelimiting enzyme in dTMP synthesis pathway [8], and thus is a potential target for drug development. Various classes of compounds, including 2,4-diaminopyrimidine, have been proposed to be effective inhibitors of Plasmodium SHMT based on binding affinity obtained from molecular docking calculations $[34,35]$. The recent study has shown that a number of 2,4-diaminopyrimidine compounds can inhibit Plasmodium SHMT [21]. Further optimization employing a target-based design approach should allow design of more effective anti-malarial drugs targeting Plasmodium SHMT.

\section{Conclusions}

Both isoforms of plasmodial SHMT are highly expressed during the trophozoite stage, which highlight the role of these enzymes during parasite growth and development.
Fluorescent-tagged plasmodial SHMT proteins confirmed the expected sub-cellular location of cytoplasmic and mitochondrial SHMT. The indispensable role of Plasmodium cSHMT for intra-erythrocytic development was inferred from the inability to generate cshmt knockout parasites, which also indicated that the two SHMT isoforms do not possess redundant function typical of other eukaryotes. Therefore, cSHMT is a validated antimalarial drug target. Furthermore, transgenic P. berghei containing human malaria cshmt in place of endogenous gene will serve as an in vivo model for evaluation of novel anti-malarials directed against human plasmodial SHMT.

\section{Additional file}

Additional file 1: Primers used in the study.

\section{Abbreviations}

SHMT: Serine hydroxymethyltransferase; DHFR: Dihydrofolate reductase; TS: Thymidylate synthase; MTHF: Methylenetetrahydrofolate;

THF: Tetrahydrofolate; c: Cytosolic; m: Mitochondrial; Pf: Plasmodium falciparum; Pb: P. berghei; PV: P. vivax; 3'UTR: 3' untranslated region; 5'UTR: 5' untranslated region; BSD: Blasticidin S deaminase; PYR: Pyrimethamine; RBC: Erythrocyte; iRBC: Infected erythrocytes; DsRed: Discosoma sp. Red fluorescent protein; gDNA: Genomic DNA.

\section{Competing interests}

The authors declare that they have no competing interests.

\section{Authors' contributions}

WP performed the study and drafted the manuscript. DK participated in the design of the study and drafted the manuscript. CU and YY discussed and commented on the manuscript. UL conceived of the study, and participated in its design and coordination and helped to draft the manuscript. All authors read and approved the final manuscript.

\section{Acknowledgements}

The study was financial supported by Cluster Program and Management Office for Discovery based Development, National Science and Technology development Agency, Thailand (CPMO-DD/P-10-11274). We thank Prof. Prapon Wilairat for editing the manuscript. We acknowledge Carl Ziess Thailand for use of Zeiss LSM 700 laser scanning confocal microscope.

Received: 28 August 2012 Accepted: 20 November 2012

Published: 22 November 2012

\section{References}

1. Sherman IW: Biochemistry of Plasmodium (malarial parasites). Microbiol Rev 1979, 43:453-495.

2. Gero AM, O'Sullivan WJ: Purines and pyrimidines in malarial parasites. Blood Cells 1990, 16:467-484.

3. Eichler $H G$, Hubbard $R$, Snell $K$ : The role of serine hydroxymethyltransferase in cell proliferation: DNA synthesis from serine following mitogenic stimulation of lymphocytes. Biosci Rep 1981, 1:101106.

4. Snell K, Natsumeda Y, Weber G: The modulation of serine metabolism in hepatoma 3924A during different phases of cellular proliferation in culture. Biochem J 1987, 245:609-612.

5. Snell K, Natsumeda Y, Eble JN, Glover JL, Weber G: Enzymic imbalance in serine metabolism in human colon carcinoma and rat sarcoma. $\mathrm{Br} J$ Cancer 1988, 57:87-90

6. Thorndike J, Gaumont Y, Kisliuk RL, Sirotnak FM, Murthy BR, Nair MG, Piper JR: Inhibition of glycinamide ribonucleotide formyltransferase and other 
folate enzymes by homofolate polyglutamates in human lymphoma and murine leukemia cell extracts. Cancer Res 1989, 49:158-163.

7. Rao NA, Talwar R, Savithri HS: Molecular organization, catalytic mechanism and function of serine hydroxymethyltransferase-a potential target for cancer chemotherapy. Int J Biochem Cell Biol 2000, 32:405-416.

8. Nirmalan N, Wang P, Sims PF, Hyde JE: Transcriptional analysis of genes encoding enzymes of the folate pathway in the human malaria parasite Plasmodium falciparum. Mol Microbiol 2002, 46:179-190.

9. Maenpuen $S$, Sopitthummakhun $K$, Yuthavong Y, Chaiyen $P$, Leartsakulpanich U: Characterization of Plasmodium falciparum serine hydroxymethyltransferase-A potential antimalarial target. Mol Biochem Parasitol 2009, 168:63-73.

10. Appling DR: Compartmentation of folate-mediated one-carbon metabolism in eukaryotes. FASEB J 1991, 5:2645-2651.

11. Gagnon D, Foucher A, Girard I, Ouellette M: Stage specific gene expression and cellular localization of two isoforms of the serine hydroxymethyltransferase in the protozoan parasite Leishmania. Mol Biochem Parasitol 2006, 150:63-71

12. Alfadhli S, Rathod PK: Gene organization of a Plasmodium falciparum serine hydroxymethyltransferase and its functional expression in Escherichia coli. Mol Biochem Parasitol 2000, 110:283-291.

13. Pang CK, Hunter JH, Gujjar R, Podutoori R, Bowman J, Mudeppa DG, Rathod PK: Catalytic and ligand-binding characteristics of Plasmodium falciparum serine hydroxymethyltransferase. Mol Biochem Parasitol 2009, 168:74-83.

14. Salcedo E, Sims PF, Hyde JE: A glycine-cleavage complex as part of the folate one-carbon metabolism of Plasmodium falciparum. Trends Parasitol 2005, 21:406-411.

15. Sopitthummakhun K, Maenpuen S, Yuthavong Y, Leartsakulpanich U, Chaiyen P: Serine hydroxymethyltransferase from Plasmodium vivax is different in substrate specificity from its homologues. FEBS J 2009, 276:4023-4036.

16. Franca TC, Pascutti PG, Ramalho TC, Figueroa-Villar JD: A three-dimensional structure of Plasmodium falciparum serine hydroxymethyltransferase in complex with glycine and 5-formyl-tetrahydrofolate. Homology modeling and molecular dynamics. Biophys Chem 2005, 115:1-10.

17. Sato S, Rangachari K, Wilson RJ: Targeting GFP to the malarial mitochondrion. Mol Biochem Parasitol 2003, 130:155-158.

18. Janse CJ, Ramesar J, Waters AP: High-efficiency transfection and drug selection of genetically transformed blood stages of the rodent malaria parasite Plasmodium berghei. Nat Protoc 2006, 1:346-356.

19. Trager W, Jensen JB: Human malaria parasites in continuous culture. Science 1976, 193:673-675.

20. Crabb BS, Triglia T, Waterkeyn JG, Cowman AF: Stable transgene expression in Plasmodium falciparum. Mol Biochem Parasitol 1997. 90:131-144.

21. Sopitthummakhun $K$, Thongpanchang $C$, Vilaivan $T$, Yuthavong $Y$, Chaiyen $P$, Leartsakulpanich U: Plasmodium serine hydroxymethyltransferase as a potential anti-malarial target: inhibition studies using improved methods for enzyme production and assay. Malar J 2012, 11:194.

22. Miao J, Li J, Fan Q, Li X, Cui L: The puffamily RNA-binding protein PfPuf2 regulates sexual development and sex differentiation in the malaria parasite Plasmodium falciparum. J Cell Sci 2010, 123:1039-1049.

23. Johnson RA, McFadden Gl, Goodman CD: Characterization of two malaria parasite organelle translation elongation factor $\mathrm{G}$ proteins: the likely targets of the anti-malarial fusidic acid. PLoS One 2011, 6:e20633.

24. Shaner NC, Steinbach PA, Tsien RY: A guide to choosing fluorescent proteins. Nat Methods 2005, 2:905-909.

25. Alberts B, Johnson A, Lewis J, Raff M, Roberts K, Walter P: Molecular Biology of the Cell. New York: Garland Science; 2002

26. Bender A, van Dooren GG, Ralph SA, McFadden Gl, Schneider G: Properties and prediction of mitochondrial transit peptides from Plasmodium falciparum. Mol Biochem Parasitol 2003, 132:59-66.

27. Read M, Muller IB, Mitchell SL, Sims PF, Hyde JE: Dynamic subcellular localization of isoforms of the folate pathway enzyme serine hydroxymethyltransferase (SHMT) through the erythrocytic cycle of Plasmodium falciparum. Malar J 2010, 9:351.

28. Janse CJ, Franke-Fayard B, Mair GR, Ramesar J, Thiel C, Engelmann S, Matuschewski K, van Gemert GJ, Sauerwein RW, Waters AP: High efficiency transfection of Plasmodium berghei facilitates novel selection procedures. Mol Biochem Parasitol 2006, 145:60-70.
29. Anderson DD, Stover PJ: SHMT1 and SHMT2 are functionally redundant in nuclear de novo thymidylate biosynthesis. PLoS One 2009, 4:e5839.

30. Leartsakulpanich U, Kongkasuriyachai D, Imwong M, Chotivanich K Yuthavong Y: Cloning and characterization of Plasmodium vivax serine hydroxymethyltransferase. Parasitol Int 2008, 57:223-228.

31. Emanuelsson $\mathrm{O}$, von Heijne G, Schneider G: Analysis and prediction of mitochondrial targeting peptides. Methods Cell Biol 2001, 65:175-187.

32. Stauffer GV, Plamann MD, Stauffer LT: Construction and expression of hybrid plasmids containing the Escherichia coli glyA genes. Gene 1981, 14:63-72.

33. Vatcher GP, Thacker CM, Kaletta T, Schnabel H, Schnabel R, Baillie DL: Serine hydroxymethyltransferase is maternally essential in Caenorhabditis elegans. J Biol Chem 1998, 273:6066-6073.

34. da Silva ML, da Silva Goncalves A, Batista PR, Figueroa-Villar JD, Pascutti PG, Franca TC: Design, docking studies and molecular dynamics of new potential selective inhibitors of Plasmodium falciparum serine hydroxymethyltransferase. Mol Simulat 2010, 36:5-14.

35. Franca TC, Wilter A, Ramalho TC, Pascutti PG, Figueroa-Villar JD: Molecular dynamics of the interaction of Plasmodium falciparum and human serine hydroxymethyltransferase with 5-formyl-6-hydrofolic acid analogues: design of new potential antimalarials. J Braz Chem Soc 2006, 17:1383-1392.

doi:10.1186/1475-2875-11-387

Cite this article as: Pornthanakasem et al:: Plasmodium serine hydroxymethyltransferase: indispensability and display of distinct localization. Malaria Journal 2012 11:387.

\section{Submit your next manuscript to BioMed Central and take full advantage of:}

- Convenient online submission

- Thorough peer review

- No space constraints or color figure charges

- Immediate publication on acceptance

- Inclusion in PubMed, CAS, Scopus and Google Scholar

- Research which is freely available for redistribution 